\title{
EMITTANCE CHARACTERIZATION OF ION BEAMS PROVIDED BY LASER PLASMA
}

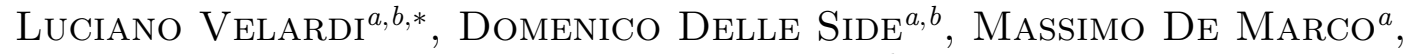 \\ VINCENZO NASSISI ${ }^{a, b}$ \\ ${ }^{a}$ Laboratorio di Elettronica Applicata e Strumentazione (LEAS), Dipartimento di Matematica e Fisica, \\ Università del Salento, Lecce, Italy \\ ${ }^{b}$ Istituto Nazionale di Fisica Nucleare (INFN) Section of Lecce, Lecce, Italy \\ * corresponding author: luciano.velardi@le.infn.it
}

\begin{abstract}
Laser ion sources offer the possibility to obtain ion beams useful for particle accelerators. Nanosecond pulsed lasers at intensities of the order of $10^{8} \mathrm{~W} / \mathrm{cm}^{2}$, interacting with solid matter in a vacuum, produce plasma of high temperature and high density. To increase the ion energy, an external post-acceleration system can be employed by means of high voltage power supplies of some tens of $\mathrm{kV}$. In this work, we characterize the ion beams provided by an LIS source and post-accelerated. We calculated the produced charge and the emittance. Applying $60 \mathrm{kV}$ of accelerating voltage and laser irradiance of $0.1 \mathrm{GW} / \mathrm{cm}^{2}$ on the $\mathrm{Cu}$ target, we obtain $5.5 \mathrm{~mA}$ of output current and normalized beam emittance of $0.2 \pi \mathrm{mm}$ mrad. The brightness of the beams was $137 \mathrm{~mA}(\pi \mathrm{mm} \mathrm{mrad})^{-2}$.
\end{abstract}

KEYWORDS: LIS source, ion beam, emittance.

\section{INTRODUCTION}

It is known that the presence of specific doped ions can significantly modify the chemical-physical properties of many materials. Today many laboratories, including LEAS, are involved in developing accelerators of very contained dimensions, easy to install in small laboratories and hospitals. The use of ion sources facilitates the improvement of ion beams of moderate energy and with good geometric qualities. They are used for the production of innovative electronic and optoelectronic films [7, biomedical materials [8, 16, new radiopharmacy applications [14, 6], hadrontherapy applications [3], and to improve the oxidation resistance of many materials [13].

There are many methods for obtaining particle beams; application of the pulsed laser ablation (PLA) technique (the technique that we adopt in this work) enables ions to be obtained solid targets, without any previous preparation, whose energy can easily be increased by post-acceleration systems [2, 12, 1]. In this way, plasma can be generated from many materials, including from refractory materials [9, 15].

In this work, we characterize the ion beams provided by a laser ion source (LIS) accelerator composed of two independent accelerating sectors, using an excimer $\mathrm{KrF}$ laser to get PLA from the pure $\mathrm{Cu}$ target. Using a home-made Faraday cup and a pepper pot system, we studied the extracted charges and the geometric quality of the beams.

\section{MATERIALS AND METHODS}

The LIS accelerator consists of a KrF excimer laser operating in the UV range to get PLA from solid targets, and a vacuum chamber device for expanding the plasma plume, extracting and accelerating its ion component. The maximum output energy of the laser is $600 \mathrm{~mJ}$. It works at $248 \mathrm{~nm}$ wavelength and the pulse duration is $25 \mathrm{~ns}$. The angle formed by the laser beam with respect to the normal to the target surface is $70^{\circ}$. During our measurements, the laser spot area onto the target surface is fixed at $0.005 \mathrm{~cm}^{2}$. The laser beam strikes the solid targets and it generates plasma in a expansion chamber (EC), Fig. 1

This chamber is closed around to the target support $(\mathrm{T})$ and it is put at a positive high voltage $(\mathrm{HV})$ of $+40 \mathrm{kV}$. The length of the expansion chamber $(18 \mathrm{~cm})$ is sufficient to decrease the plasma density [5]. The plasma expands inside the EC and, since there is no electric field, breakdowns are absent. Thanks to the plasma expansion, the charges reach the extremity of the expansion chamber, which is drilled with a $1.5 \mathrm{~cm}$ hole to allow ion extraction. A pierced ground electrode (GE) is placed at $3 \mathrm{~cm}$ distance from EC. In this way it is possible to generate an intense accelerating electric field between EC and GE. Four capacitors of $1 \mathrm{nF}$, between EC and ground, stabilize the accelerating voltage during fast ion extraction.

After GE, a third electrode (TE) is placed $2 \mathrm{~cm}$ from it, and it is connected to a power supply of negative bias voltage $-20 \mathrm{kV}$. It is also utilized as a Faraday cup collector.

TE is connected to the oscilloscope by an HV capacitor $(2 \mathrm{nF})$ and a voltage attenuator, $\times 20$, in order to separate the oscilloscope from the $\mathrm{HV}$ and to suit the electric signal to the oscilloscope input voltage. The value of the capacitors $(4 \mathrm{nF})$ applied to stabilize the accelerating voltage and the value 


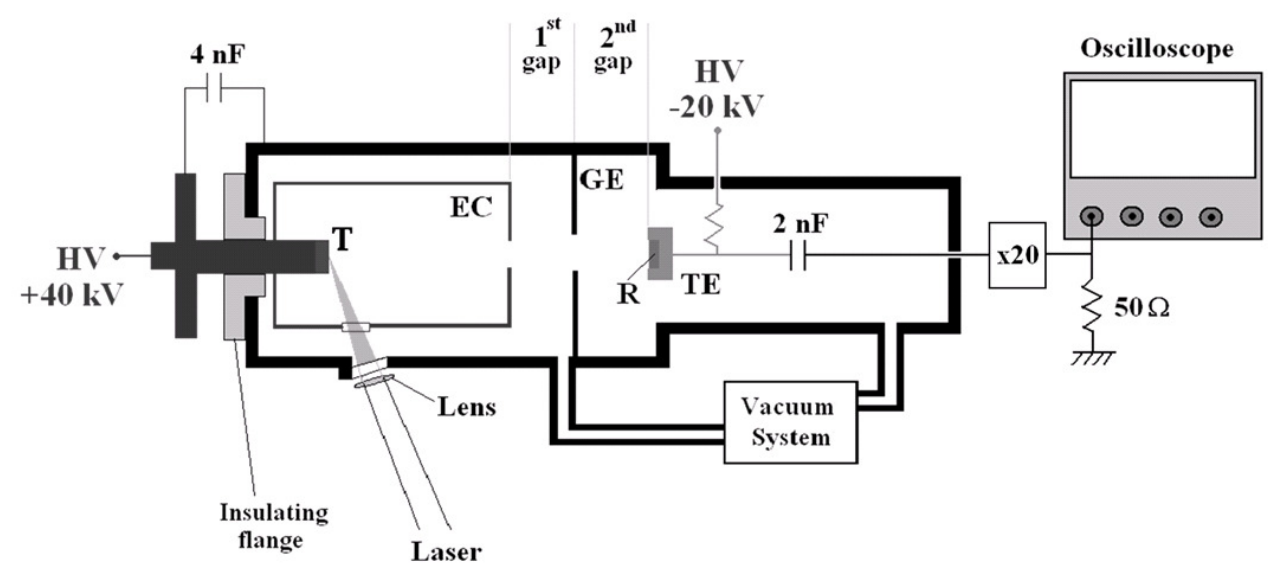

FiguRE 1. Schematic drawing of the LIS accelerator (T: Target support, EC: Expansion Chamber, GE: Ground Electrode, R: Radiochromic, TE: Third Electrode).

of the capacitors $(2 \mathrm{nF})$ used to separate the oscilloscope from the $\mathrm{HV}$ are calculated assuming a storage charge higher that the extracted one. Under this condition, the accelerating voltages during charge extraction are constant and the oscilloscope is able to record the real signal.

TE is not able to support the suppressing electrode on the cup collector, and secondary electron emission, caused by high ion energy, is therefore present. In this configuration, we are aware that the output current values are about $20 \%$ higher than the real values [11].

\section{Results AND DisCUsSiOn}

The value of the laser irradiance used to produce the ion beams was $1.0 \times 10^{8} \mathrm{~W} \mathrm{~cm}^{-2}$ and the ablated target was a pure $(99.99 \%)$ disk of $\mathrm{Cu}$.

Figure 2 shows the time of flight (TOF) spectra obtained at 60,40 and $30 \mathrm{kV}$ of total accelerating voltage from the $\mathrm{Cu}$ target, detecting the ion emission with the Faraday cup placed $23 \mathrm{~cm}$ from the target. The vertical axis represents the output current. The maximum output current is reached applying 40 and $20 \mathrm{kV}$, respectively, on the first gap (EC-GE) and on the second gap (GE-TE).

The space charge effects are more evident at the lowest accelerating voltage applied on the first gap, although they are present anyway. So, considering only the TOF curves out of the charge domination effects, we obtain the behaviour of the accelerated charge with respect to the accelerating voltage. From these results, we can observe the absence of a saturation phase. In fact the curves in Fig. 3 have a growing trend with respect to the applied voltage. The growing trend on the first gap voltage is larger than the one dependent on the second gap voltage. Theoretically, we can expect to observe a constant trend for the curves, dependent principally on the voltages of the second acceleration gap, because the charge is already extracted and its value should be constant. We can as-

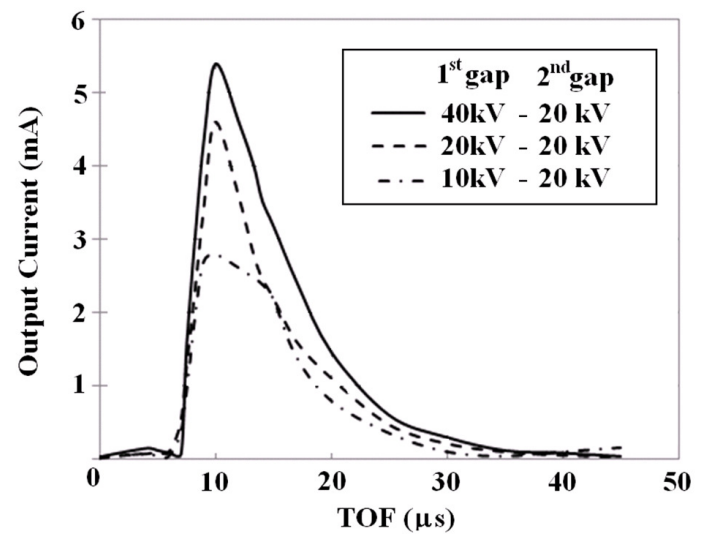

Figure 2. Waveforms of the output current at different accelerating voltages for a $\mathrm{Cu}$ target; laser irradiance: $0.1 \mathrm{GW} \mathrm{cm}^{-2}$.

cribe this behaviour toi the secondary emission of electrons from the cup collector, because we are not able to prevent this emission, owing to the absence of a suppressing electrode on the Faraday cup. So we expect that the real charge must be increased by about $20 \%$ for the used voltage values.

Fixing the voltage at $20 \mathrm{kV}$ in the second gap, the extraction current increased with the change of the voltage applied on first gap, reaching $150 \%$ at the maximum voltage of $40 \mathrm{kV}$ with respect to the value obtained at $0 \mathrm{kV}$. This result implies strong dependence of the extraction efficiency on the first stage voltage. In effect, simulations results preformed in previous works, have shown, tha the electric field strenght near the EC hole increased with respect to the applied voltage. This fact can enlarge the extracting volume inside the anode, and as a consequence, the extraction efficiency. The $\mathrm{Cu}$ target at zero voltage produced ion beams containing $1.2 \times 10^{11}$ ions/pulse $\left(0.7 \times 10^{11}\right.$ ions $\left.^{-2}\right)$. Instead, applying accelerating voltages of 40 and $20 \mathrm{kV}$ in the first and sec- 


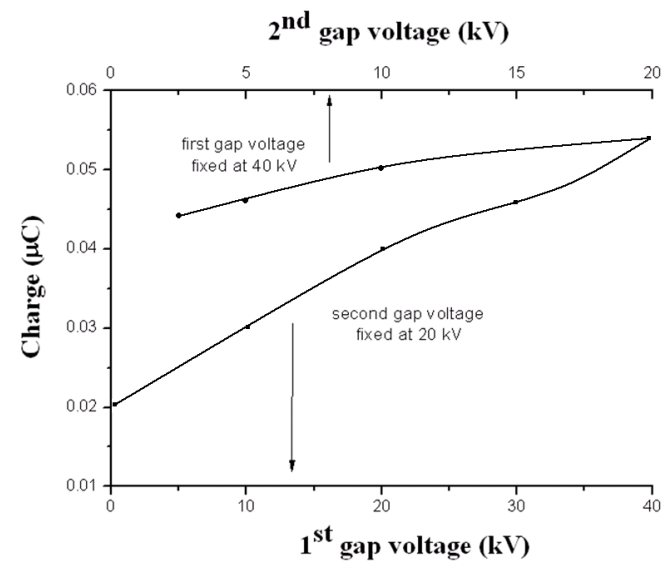

Figure 3. Output charge on accelerating voltages for the $\mathrm{Cu}$ target; laser irradiance: $0.1 \mathrm{GW} \mathrm{cm}^{-2}$.

ond accelerating gap, respectively, we obtained an increase of the ion dose up to $3.4 \times 10^{11}$ ions/pulse,

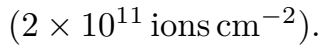

For a geometric characterization of the beam, we performed emittance measurements by the pepper pot technique. We can assume the propagation direction of the beam along the $z$ axis. So the $x$-plane emittance $x$ is $1 / \pi$ times the area $A_{x}$ in the $x x^{\prime}$ trace plane $\left(\mathrm{TP}_{x}\right)$ occupied by the points representing the beam particles at a given value of $z$, namely

$$
\varepsilon_{x}=\frac{A_{x}}{\pi} .
$$

In the phase plane $\left(\mathrm{PP}_{x}\right)$, the area of the particle is defined as [1]

$$
A_{x}^{0}=\iint_{f_{2}^{0} \neq 0} \mathrm{~d} x \mathrm{~d} p_{x}=m_{0} c \beta \gamma A_{x},
$$

where $f_{2}^{0}$ is the distribution function of the particles, $m_{0}$ is the rest mass of the particles, $\beta=v / c$ and $\gamma=1 /\left(1-\beta^{2}\right)^{1 / 2}$.

Actually, it is necessary to define an invariant quantity of the motion called normalized emittance $\varepsilon_{\mathrm{n} x}$ in the $\mathrm{TP}_{x}$. Therefore, by Liouville's theorem, it is known that the area occupied by the particle beam in $\mathrm{PP}_{x}$ is an invariant quantity and the normalised emittance can assume the form

$$
\varepsilon_{\mathrm{n} x}=\beta \gamma \varepsilon_{x} .
$$

Figure 4 shows a sketch of the system used to measure the emittance value.

The mask we used has 5 holes of $1 \mathrm{~mm}$ in diameter, and it was fixed on the GE. One hole is in the centre of the mask and 4 holes are at $3.5 \mathrm{~mm}$ from the centre. We used Gafchromic EBT radiochromic films (R) as photo-sensible screen, placed on the TE.

Radiochromic detectors take advantage of the direct impression of a material by the absorption of energetic radiation, without requiring latent chemical, optical, or thermal development or amplification. A radiochromic film changes its optical density as a function of the absorbed dose. This property, and the relative ease of use, led to the adoption these detectors as simple ion beam transverse properties diagnostic tools.

So, the ion beam after the mask imprinted the radiochromic film and then it was possible to measure the divergence of all beamlets. The divergence values allowed us to determine the beam area $A_{x}$ in $\mathrm{TP}_{x}$. We applied 250 laser shots to imprint the radiochromic films.

We measured the emittance for different accelerating voltage values. We fixed the accelerating voltage of the second gap at $20 \mathrm{kV}$, while the accelerating voltage of the first gap was put at 10,20 and $40 \mathrm{kV}$. So, the obtained values of the area in the $\mathrm{TP}_{x}$ resulted in 613,545 and $435 \mathrm{~mm} \mathrm{mrad}$ for 30,40 and $60 \mathrm{kV}$ of total accelerating field, respectively (Fig. 5 ).

Considering Eq. 3, we found the normalized emittance values. For all the applied voltage values, the normalized emittance resulted constant: $\varepsilon_{\mathrm{n} x}=0.2 \pi \mathrm{mm}$ mrad.

Therefore, to estimate the total properties of the delivered beams, it is necessary to introduce the concept of brightness. Brightness is the ratio between the current and the emittance along the $x$ - and the $y$-axis. Generally assuming $x$ equal to $y$, the normalised brightness becomes

$$
B_{\mathrm{n}}=\frac{I}{\varepsilon_{\mathrm{n} x}^{2}}
$$

By Eq. 4 at the current peak $(5.5 \mathrm{~mA})$, the brightness values resulted in $137 \mathrm{~mA}(\pi \mathrm{mm} \mathrm{mrad})^{-2}$ at $60 \mathrm{kV}$ of accelerating voltage.

Due to low emittance and high current, this apparatus is very promising for use for feeding large accelerators. The challenge of the moment is to get accelerators with dimensions so small that can be easily deployed in small laboratories and hospitals.

\section{Conclusions}

Post-acceleration of ions emitted from laser-generated plasma can be developed to obtain small and compact accelerating machines. The output current can easily increase on accelerating voltage. The applied voltage can cause breakdowns, and for this reason the design of the chamber is very important (primarily its dimensions and morphology). We have also shown that two gaps of acceleration can efficiently increase the ion energy. By increasing the voltage of the first accelerating gap, we substantially increased the efficiency of the extracted current due to the rise of the electric field and the extracting volume inside the EC. The charge extracted without the electric fields was $0.7 \times 10^{11}$ ions $/ \mathrm{cm}^{2}$. At the maximum accelerating voltage the ion dose was $2 \times 10^{11}$ ions $/ \mathrm{cm}^{2}$, and the corresponding peak current was $5.5 \mathrm{~mA}$. We 


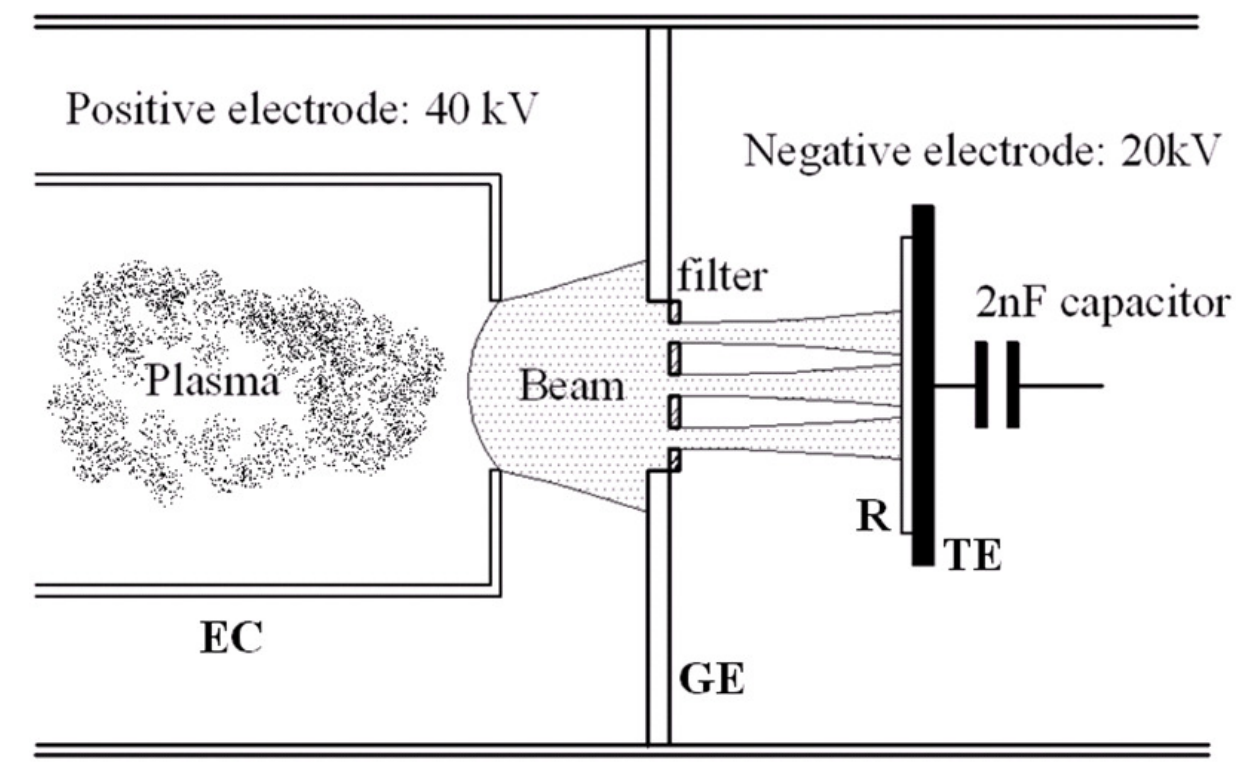

FiguRE 4. Sketch of the pepper pot system used to measure the emittance.

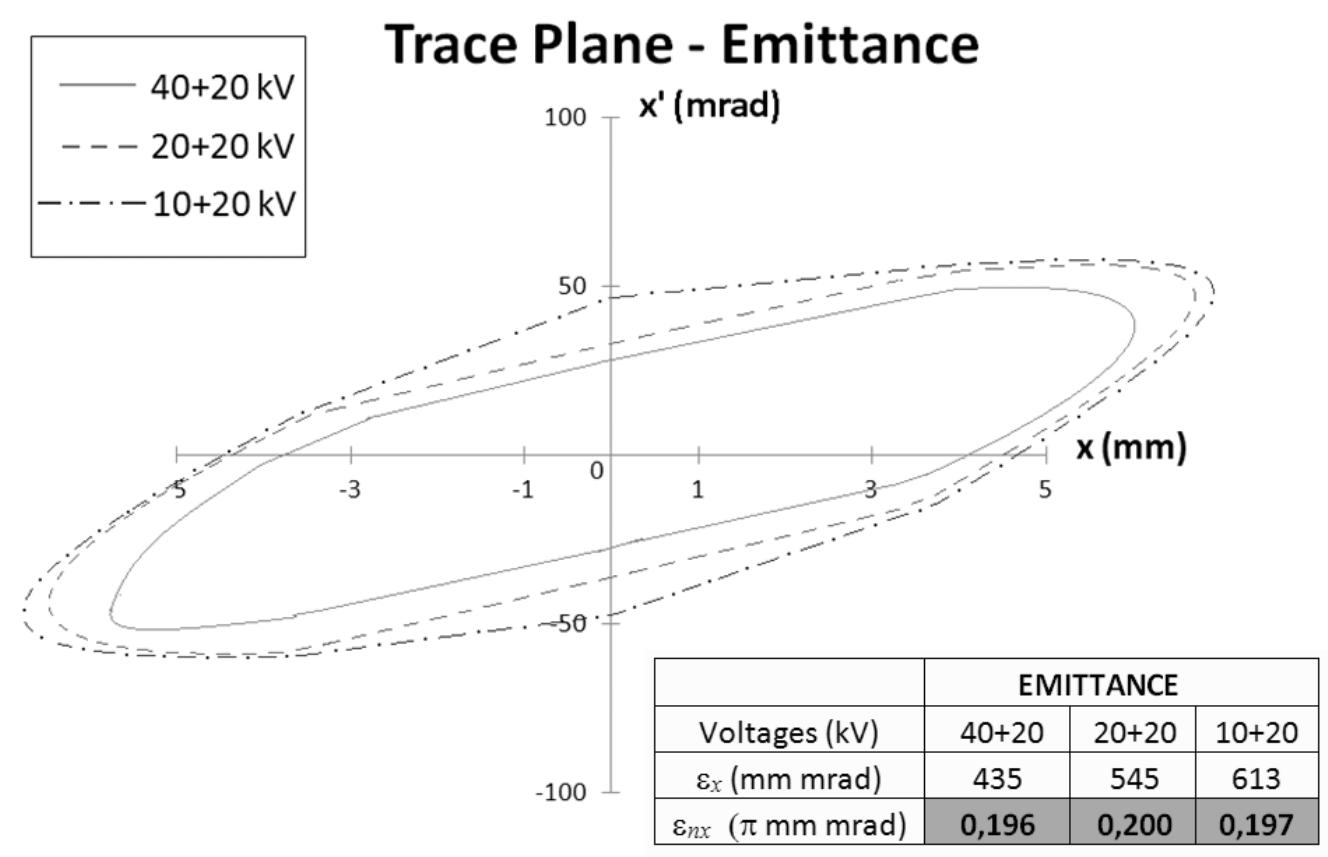

FIGURE 5. Emittance diagram in the trace plane for different accelerating voltage values. 
measured the geometric characteristics of the beam utilising the pepper pot method. We found a low value for the normalized emittance of our beams, $\varepsilon_{\mathrm{n} x}=0.2 \pi \mathrm{mm}$ mrad. The resulting brightness values were therefore $137 \mathrm{~mA}(\pi \mathrm{mm} \mathrm{mrad})^{-2}$.

This study has demonstrated that our apparatus can produce ion beams of good quality, e.g. with a low emmitance value and high current. For this reason it is very promising for use in feeding large accelerators.

\section{ACKNOWLEDGEMENTS}

The work was supported by the Fifth National Committee of INFN in the LILIA experiment.

\section{REFERENCES}

[1] F. Belloni, D. Doria, A. Lorusso, A. Nassisi. Study of particle acceleration of $\mathrm{Cu}$ plasma. Rev Sci Instrum 75(11):4763-4768, 2004.

[2] A. Beloglazov, V. Nassisi, M. Primavera. Excimer laser induced electron beams on an Al target: Plasma effect in a "nonplasma" regime. Rev Sci Instrum 66(7):3883-3887, 1995.

[3] F. Ciocci, F. Della Valle, A. Doria, et al. Spectroscopy of muonic hydrogen with a compact FEL. In Proc. EPAC '94, pp. 864-866. 1994.

[4] F. Belloni D. Bleiner, A. Bogaerts, V. Nassisi. Laser-induced plasmas from the ablation of metallic targets: The problem of the onset temperature, and insights on the expansion dynamics. J Appl Phys 101(8):83301, 2007.

[5] D. Doria, F. Belloni, A. Lorusso, et al. Recombination effects during the laser-produced plasma expansion. Rad Eff Def Solids 160(10-12):663-668, 2005.

[6] A. Fortin, F. Marion, B. L. Stansfield, et al. Efficiency of plasma-based ion implantation of radioisotopes $\left({ }^{32} \mathrm{P}\right)$. Surf and Coatings Technology 200(1-4):996-999, 2005.
[7] A. Lorusso, V. Nassisi, L. Velardi, G. Congedo. Si nanocrystals formation by a new ion implantation device. Nucl Instrum Meth B 266(10):2486-2489, 2008.

[8] A. Lorusso, L. Velardi, V. Nassisi, et al. Characteristic modification of UHMWPE by laser-assisted ion implantation. Rad Eff Def Solids 163(4-6):447-451, 2008.

[9] A. Lorusso, L. Velardi, V. Nassisi, et al. Polymer processing by a low energy ion accelerator. $\mathrm{Nucl}$ Instrum Meth B 266(10):2490-2493, 2008.

[10] A. Luches, M. Martino, V. Nassisi, et al. Generation of self-pulsed multiple charged ions by an $\mathrm{XeCl}$ excimer laser. Nucl Instrum Methods Phys Res A 322(2):166-169, 1992.

[11] V. Nassisi, E. Giannico. Characterization of high charge electron beams induced by excimer laser irradiation. Rev Sci Instrum 70(8):3277-3281, 1999.

[12] V. Nassisi, A. Pedone. Physics of the expanding plasma ejected from a small spot illumined by an ultraviolet pulsed laser. Rev Sci Instrum 74(1):68-72, 2003.

[13] F. Noli, A. Lagoyannis, P. Misaelides. Oxidation resistance of Y-implanted steel using accelerator based techniques. Nucl Instrum Methods Phys Res B 266(10):2437-2440, 2008.

[14] H. Takeda, J. H. Billen, S. Nath, et al. A compact high-power proton linac for radioisotope production. In Proc. IEEE Part. Accel. Conf., vol. 2, pp. 8006-8013. 1995.

[15] L. Torrisi, F. Caridi, L. Giuffrida. Comparison of Pd plasmas produced at $532 \mathrm{~nm}$ and $1064 \mathrm{~nm}$ by a Nd:YAG laser ablation. Nucl Instr and Methods in Physics Res B 268(13):2285-2291, 2010.

[16] Z. Yu. Study on the interaction of low-energy ions with organisms. Surface $\&$ Coatings Technology 201(19-20):8006-8013, 2007. 